\title{
CONCENTRACIONES DE YODO EN ORINA Y EN SAL DE CONSUMO EN MUJERES ENTRE 12 A 49 AÑOS DEL PERÚ
}

\author{
Carolina Tarqui-Mamani1,2,a, José Sánchez-Abanto ${ }^{1, b}$, Doris Alvarez-Dongo ${ }^{1, c}$, Teresa Jordán-Lechuga ${ }^{1, d}$, \\ Inés Fernández-Tinco ${ }^{1, d}$
}

\begin{abstract}
RESUMEN
Objetivos. Determinar las concentraciones de yodo en orina y sal de consumo en mujeres entre 12 a 49 años en Perú. Materiales y métodos. Se realizó un estudio observacional y transversal. Durante el 2012 y 2013 , se incluyó mujeres entre 12 a 49 años residentes en los hogares peruanos seleccionadas mediante un muestreo probabilístico, estratificado y multietápico. La determinación de yodo en orina se realizó por espectrofotometría basada en la reacción de SandellKolthoff. La evaluación cualitativa de yodo en sal se realizó por yoditest y la cuantitativa por volumetría. El procesamiento se realizó mediante muestras complejas con ponderaciones. Se obtuvo medianas, rango intercuartílico y percentiles. Resultados. La mediana de yoduria en las participantes fue 250,4 ug/L; los departamentos con medianas de yoduria elevadas fueron: Moquegua (389,3 ug/L); Tacna (320,5 ug/L); Madre de Dios (319,8 ug/L), y Ucayali (306,0 ug/L); mientras que Puno (192,9 ug/L); Piura (188,1 ug/L) y Tumbes (180,5 ug/L) tuvieron medianas dentro de lo recomendado por la OMS. La mediana de yoduria en gestantes fue 274,6 ug/L (RIQ: $283 \mathrm{ug} / \mathrm{L}$ ). El 82,5\% de las muestras de sal tuvieron yodo $\geq 30$ ppm y $1,9 \%$ tuvo valores de 0 ppm. Conclusiones. La mediana de yoduria en las mujeres peruanas está por encima de lo recomendado por la OMS y la mayoría de las muestras de sal tuvieron concentraciones adecuadas de yodo según la OMS.
\end{abstract}

Palabras clave: Yodo; Deficiencia de yodo; Encuestas nutricionales; Epidemiología (fuente: DeCS BIREME).

\section{IODINE CONCENTRATION IN URINE AND SALT CONSUMPTION IN WOMEN BETWEEN 12 TO 49 YEARS OF AGE IN PERU}

\begin{abstract}
Objectives. To determine the concentrations of iodine in urine and salt intake in women between 12-49 years of age in Peru. Materials and methods. An observational and cross-sectional study was performed. During 2012 and 2013 , the study included women aged between 12-49 years living in Peruvian households selected through a probabilistic, stratified and multistage sample. The determination of iodine in urine was performed based on the Sandell-Kolthoff reaction spectrophotometry. The qualitative evaluation of iodine salt was performed by Yoditest and the quantitative by volumetric analysis. Processing was performed using complex samples with weights. Medians, interquartile range and percentiles were obtained. Results. The median urinary iodine levels in the participants was $250.4 \mathrm{ug} / \mathrm{L}$. The regions with elevated median urinary iodine levels were: Moquegua (389.3 ug/L); Tacna (320.5 ug/L); Madre de Dios (319.8 ug/L), and Ucayali (306.0 ug/L); while Puno (192.9 ug/L); Piura (188.1 ug/L) and Tumbes (180.5 ug/L) had medians within ranges recommended by the WHO. The median urinary iodine in pregnant women was $274.6 \mathrm{ug} / \mathrm{L}$ (IQR: $283 \mathrm{ug} / \mathrm{L}$ ). $82.5 \%$ of the samples had iodine salt $\geq 30 \mathrm{ppm}$ and $1.9 \%$ had values of $0 \mathrm{ppm}$. Conclusions. The median urinary iodine in Peruvian women is higher than recommended by the WHO and the majority of the samples had adequate iodine concentrations according to $\mathrm{WHO}$ recommendations.
\end{abstract}

Key words: lodine; lodine deficiency; Nutritional surveys; Epidemiology (source: MeSH NLM).

\section{INTRODUCCIÓN}

El yodo es un oligoelemento esencial para la síntesis de las hormonas tiroideas, triyodotironina (T3) y tiroxina (T4) y contribuye en la síntesis de las hormonas tiroideas que participan en el desarrollo cerebral y regulación del metabolismo ${ }^{(1)}$. Una dieta deficiente en yodo se asocia con diversas enfermedades, que se conoce como desórdenes por deficiencia de yodo (DDY) ${ }^{(2)}$. La deficiencia de yodo es la principal causa en el mundo de retraso mental que puede ser prevenible (3), así mismo, produce alteraciones neurointelectuales (la forma más severa es el cretinismo

\footnotetext{
Centro Nacional de Alimentación y Nutrición, Instituto Nacional de Salud. Lima, Perú

Departamento de Medicina Preventiva y Salud Pública, Facultad de Medicina, Universidad Nacional Mayor de San Marcos. Lima, Perú.

a Doctora en Salud Pública, magíster en Epidemiología con especialidad en Estadística; ${ }^{\mathrm{b}}$ nutricionista magíster en nutrición; ${ }^{\mathrm{c}}$ nutricionista; ${ }^{\mathrm{d}}$ bióloga. Recibido: : 01-08-2014 Aprobado: 08-04-15
} 
y la más leve el deterioro intelectual); es especialmente dañino durante las primeras etapas del embarazo y en la primera infancia. Por otro lado, una alta ingesta de yodo podría producir cambios en los niveles de hormonas tiroideas y potenciales cambios en la prevalencia de disfunción tiroidea como el de hipertiroidismo inducido por yodo ${ }^{(4)}$. Las diversas intervenciones implementadas para incrementar el consumo de yodo en la población han contribuido en la disminución del bocio y los DDY, en general. Sin embargo, se ha observado que podría existir una sobreingesta del yodo requerido, lo cual se convierte en un nuevo problema de salud pública debido al hipertiroidismo inducido por yodo ${ }^{(5-7)}$.

La carencia de yodo es la mayor causa individual en el mundo de retraso mental susceptible de prevención mediante la fortificación de la sal con yodo. Se estima que el costo anual de la yodación de la sal es aproximadamente USD 0,02-0,05 por niño protegido, y el costo por muerte evitada de un niño por años de vida ajustados por discapacidad (AVAD) sería de USD 1000; mientras que por años de vida ganado ajustado por discapacidad sería USD 34-36,1 ${ }^{(8,9)}$. El Banco Mundial ha calculado que cada dólar dedicado a la prevención de los trastornos por deficiencia de yodo conlleva una ganancia en productividad de USD 28,00, por tanto, la eliminación de la carencia de yodo constituye una de las intervenciones más rentables que contribuye a incrementar la productividad ${ }^{(10)}$.

A comienzos del siglo XX, los desórdenes por deficiencia de yodo fueron reconocidos como un problema de salud pública en la mayoría de países de América. La Organización Mundial de la Salud (OMS) estimó que 110 países fueron afectados por el bocio a consecuencia de la deficiencia de yodo (11). Del 2003 al 2011, el número de países con deficiencia de yodo disminuyeron de 54 a 32 y el número de países con adecuada ingesta de yodo se incrementó de 67 a 105,9 (12); paralelamente, $71 \%$ de la población tenía acceso a la sal yodada, cifra que denota un incremento con respecto a 1990, que fue aproximadamente $20 \%{ }^{(12)}$. La OMS-UNICEF, recomiendan que la ingesta diaria de yodo en preescolares ( 0 a 59 meses) sea de $90 \mu \mathrm{g}$, en escolares (6 a 12 años); de $120 \mu \mathrm{g}$; en adolescentes y adultos (por encima de 12 años) de $150 \mu \mathrm{g}$, mientras que en gestantes y lactantes de $250 \mu g^{(13)}$.

El Perú es uno de los países que ha trabajado arduamente en la lucha contra el bocio. El Ministerio de Salud del Perú (MINSA) asumió la vigilancia de los desórdenes por deficiencia de yodo, inicialmente se encontró que el $87 \%$ de los pueblos de la sierra y de la selva presentaban bocio endémico. La prevalencia promedio de bocio en escolares fue del orden del $36 \%$ y la mediana del yodo urinario estuvo en $74 \mathrm{ug} / \mathrm{L}$ (normal $>100 \mathrm{ug} / \mathrm{L}$ ) ${ }^{(14)}$. Existen algunos estudios realizados en submuestras o muestras relativamente pequeñas, que reportan que la mediana de yoduria en mujeres en edad fértil fue $266 \mu \mathrm{g} / \mathrm{L}$; y la proporción de hogares con ingesta de sal con presencia de yodo $\geq 7 \mathrm{ppm}$ fue $97,5 \%$ y $\geq 15 p p m$ fue $85,0 \%{ }^{(6)}$, otros estudios reportan que la mediana de yoduria en los escolares entre 6 a 11 años de escuelas públicas está por encima de lo recomendado por la OMS ${ }^{(7)}$.

A juzgar por los reportes previos, se estaría frente a un nuevo problema de salud pública debido a una ingesta excesiva de yodo proveniente de diversas fuentes de yodo, como la sal fortificada, alimentos fortificados, productos marinos, vegetales, entre otros, por ello, es imperante, realizar la evaluación de yodo en orina y sal de consumo en una muestra suficiente que permita tener indicadores con inferencia nacional; con información real que sirva para fortalecer, replantear estrategias o intervenciones que contribuya a mejorar la salud de la población. El objetivo del estudio fue determinar las concentraciones de yodo en orina y en sal de consumo, en mujeres entre 12 a 49 años y gestantes en el Perú.

\section{MATERIALES Y MÉTODOS}

\section{DISEÑO Y POBLACIÓN DE ESTUDIO}

Estudio observacional y transversal, la recolección de datos se realizó durante el 2012-2013. Se incluyó a mujeres entre 12 a 49 años y gestantes que residen en los hogares peruanos, se excluyó a las mujeres con hipertiroidismo, menstruación, medicación de yodo por problemas tiroideos o que estén tomando suplemento de sulfato ferroso.

Se realizó un muestreo probabilístico, estratificado y multietápico e independiente en cada departamento del Perú (15). Para el cálculo del tamaño de la muestra por departamento se evalúa la precisión estadística obtenida en la muestra de la Encuesta Nacional de Hogares (ENAHO) 2006 (15) para estimar algunas características de la vivienda y del hogar, características de los miembros del hogar, educación, salud, empleo, ingreso y gastos del hogar; nivel de confianza al 95\% y precisión $5 \%$. También se consideró el efecto de diseño y las tasas de no respuestas para realizar los ajustes respectivos. La muestra incluyó 1000 conglomerados, 6622 viviendas (689 en el área urbana y 311 en la rural) distribuidos en el Perú. Cabe precisar, que el estudio se realizó en el marco del convenio entre el Instituto Nacional de Salud (INS) y el Instituto Nacional de Estadística e Informática (INEI), mediante el cual, el Centro Nacional de Alimentación y Nutrición (CENAN) utiliza la muestra 
ENAHO en su totalidad para la evaluación de los indicadores nutricionales y es independiente al estudio del ENAHO que desarrolla el INEI anualmente.

\section{DETERMINACIÓN DE YODURIA}

Se solicitó a todas las participantes la primera muestra de orina del día, previamente, se entregó un frasco de $30 \mathrm{~mL}$ de cierre hermético, rotulado. Las muestras fueron depositadas en cajas con refrigerantes conservando la cadena de frio, una vez congeladas, fueron enviadas al CENAN. El análisis de las muestras se realizó mediante el método de espectrofotometría que utiliza el persulfato de amonio, y se basa en la reacción de Sandell-Kolthoff $(16,17)$ en los laboratorios de la Dirección Ejecutiva de Ciencia y Tecnología de Alimentos (DECYTA) certificado por Centers for Disease Control and Prevention (CDC). La mediana de yodo urinario de 100 a 199 ug/L se consideró niveles adecuados para las mujeres entre 12 a 49 años. La mediana de yodo urinario 150 a 249 ug/L se consideró adecuada para las gestantes.

\section{DETERMINACIÓN DE YODO EN SAL}

La evaluación semicuantitativa de yodo en sal se realizó utilizando una prueba rápida basada en almidón (Yoditest ${ }^{\circledR}$ ); para lo cual se recolectó una muestra de sal que se emplea para preparar los alimentos en el hogar y se agregó el reactivo, seguidamente, se observó el color resultante de la reacción y se comparó con una escala de colores, se registró la puntuación correspondiente en partes por millón -ppm- (0; 7; 15 y >30 ppm). Se consideró como adecuado nivel de yodo para consumo cuando se encontró valores $>30$ ppm.

La evaluación cuantitativa de yodo en sal se realizó mediante el método de volumetría ${ }^{(13)}$ en los laboratorios de la Dirección Ejecutiva de Ciencia y Tecnología de Alimentos (DECYTA), para lo cual se recolectó $120 \mathrm{~g}$ de sal (cinco cucharadas) en una bolsa de polipropileno de cierre hermético de 10 x $10 \mathrm{~cm}$ (bolsa Ziplock). Se rotuló la muestra de sal, y se envió al CENAN. En todos los casos, se registró la marca de sal. La OMS indica que la sal de consumo humano debería contener entre 20 a $40 \mathrm{mg} / \mathrm{kg}^{(18)}$, sin embargo, la normatividad peruana establece que el contenido de yodo en sal debe ser de 30 a $40 \mathrm{mg} / \mathrm{kg}^{(19,20)}$.

\section{ANÁLISIS ESTADÍSTICO}

El procesamiento se realizó mediante análisis para muestras complejas con las respectivas ponderaciones en SPSS v 22. Se calculó medianas, rango intercuartílico, percentiles, Kolmogorv Smirnov y Kruskal Wallis, se estableció como nivel de significancia $p<0,05$.

\section{CONSIDERACIONES ÉTICAS}

A todas las participantes residentes en los hogares de la muestra se les solicitó el consentimiento informado por escrito; se empleó un código identificador para el análisis de la yoduria y así garantizar la confidencialidad de los participantes. El estudio se realizó en el marco de la Vigilancia por Desórdenes de Yodo que periódicamente efectúa la Dirección Ejecutiva de Vigilancia Alimentaria y Nutricional del INS y está aprobado en el Plan Operativo Institucional.

\section{RESULTADOS}

Se evalúo a 4188 mujeres entre 12 a 49 años residentes en los hogares peruanos. El $77,1 \%$ de las mujeres residían en zona urbana: $2,1 \%$ fueron analfabetas: $23,2 \%$ tenían nivel primario: $49,2 \%$ secundaria y $25,6 \%$ superior. El 33,5\% vivían en la Lima Metropolitana: $24,3 \%$ en la costa: $31,3 \%$ en la sierra y $10,8 \%$ en la selva, por otro lado, 5,6\% estuvieron en la condición de pobreza extrema: $20,0 \%$ fueron pobres y $74,4 \%$ no pobres.

A nivel nacional, la mediana de yoduria fue más que adecuado (250,4 ug/L; RIQ: 232,51) y la mediana de yoduria en gestantes fue 274,6 $\mu \mathrm{g} / \mathrm{L}$ (RIQ: 283); considerado por encima de lo requerido.

Tabla 1. Características en la distribución de la mediana de yoduria en mujeres. Perú; 2012-2013

\begin{tabular}{|c|c|c|c|c|c|}
\hline Características & $\mathbf{N}$ & Mediana & P25 & P75 & Valor $p$ \\
\hline \multicolumn{6}{|l|}{ Grupo etario } \\
\hline 12 a 19 & 1261 & 263,6 & 160,3 & 413,2 & $<0,001$ \\
\hline 20 a 29 & 906 & 262,8 & 162,7 & 380,6 & \\
\hline 30 a 49 & 2021 & 235,1 & 142,6 & 361,5 & \\
\hline \multicolumn{6}{|l|}{ Nivel educativo } \\
\hline Analfabeto & 136 & 201,6 & 110,4 & 364,0 & $<0,001$ \\
\hline Primaria & 1185 & 246,0 & 142,4 & 402,9 & \\
\hline Secundaria & 1885 & 254,8 & 156,9 & 389,7 & \\
\hline Superior & 982 & 247,5 & 155,3 & 363,9 & \\
\hline \multicolumn{6}{|l|}{ Área } \\
\hline Urbano & 2619 & 259,8 & 161,6 & 389,7 & $<0,001$ \\
\hline Rural & 1569 & 222,0 & 124,4 & 361,4 & \\
\hline \multicolumn{6}{|l|}{ Dominios } \\
\hline Costa norte & 582 & 236,3 & 148,5 & 405,0 & $<0,001$ \\
\hline Costa centro & 389 & 250,6 & 164,9 & 377,5 & \\
\hline Costa sur & 266 & 319,6 & 204,8 & 484,3 & \\
\hline Sierra norte & 298 & 201,2 & 105,2 & 319,5 & \\
\hline Sierra centro & 885 & 250,8 & 146,1 & 383,5 & \\
\hline Sierra sur & 490 & 233,7 & 140,6 & 352,6 & \\
\hline Selva & 802 & 240,7 & 138,6 & 368,0 & \\
\hline Lima Metropolitana & 476 & 269,5 & 172,7 & 396,3 & \\
\hline \multicolumn{6}{|l|}{ Pobreza } \\
\hline Pobre extremo & 303 & 242,9 & 135,5 & 387,7 & $<0,001$ \\
\hline Pobre & 886 & 237,9 & 138,2 & 393,7 & \\
\hline No pobre & 2999 & 254,7 & 156,7 & 380,0 & \\
\hline
\end{tabular}


La distribución de la mediana de la yoduria, según características de las mujeres entre 12 a 49 años, se presenta en la Tabla 1. La mediana de yoduria fue mayor en los departamentos de Moquegua (389,3 ug/L), Tacna (320,5 ug/L), Madre de Dios (319,8 ug/L) y Ucayali (306,0 ug/L). Los departamentos que tuvieron medianas de yoduria dentro de los niveles recomendados por la OMS fueron: Puno (192,9 ug/L), Piura (188,1 ug/L) y Tumbes (180,5 ug/L); ningún departamento tuvo medianas inferiores al rango recomendado por la OMS. El resto de departamentos tuvo medianas por encima de $200 \mathrm{ug} / \mathrm{L}$ y por debajo de $300 \mathrm{ug} / \mathrm{L}$.

La distribución de yodo en sal de consumo según dominios geográficos se presenta en la Tabla 2. A nivel nacional, la evaluación cualitativa de yodo en sal de consumo mostró que el $82,5 \%$ de la sal tuvo valores por encima de $30 \mathrm{ppm}$ y $1,9 \%$ tuvo valores de $0 \mathrm{ppm}$. Del total de mujeres evaluadas que tuvieron bajo nivel de yoduria, se observó que el $11,2 \%$ tuvieron niveles de yodo en sal de consumo por debajo de 15 ppm.

Las medianas de yodo en sal de consumo, según marcas mas consumidas en Cajamarca, Huancavelica, Huánuco, Loreto y San Martín, se presentan en la Tabla 3. La marca de sal más consumida fue Emsal $(46,7 \%)$ y Marina $(23,6 \%)$ por las mujeres entre 12 a 49 años a nivel nacional. La evaluación cuantitativa de sal

Tabla 2. Distribución de yodo en sal de consumo según dominios geográficos. Perú; 2012-2013

\begin{tabular}{|c|c|c|c|c|c|}
\hline Características & $\mathbf{N}$ & $0 \mathrm{ppm}$ & $\geq 7 \mathrm{ppm}$ & $\geq 15 p p m$ & $\geq 30 \mathrm{ppm}$ \\
\hline \multicolumn{6}{|l|}{ Grupo etario } \\
\hline 12 a 19 & 1260 & 2,5 & 3,0 & 13,8 & 80,8 \\
\hline 20 a 29 & 899 & 1,8 & 2,7 & 12,6 & 83,0 \\
\hline 30 a 49 & 2016 & 1,6 & 2,5 & 12,6 & 83,3 \\
\hline \multicolumn{6}{|l|}{ Nivel educativo } \\
\hline Analfabeto & 136 & 2,7 & 6,9 & 14,7 & 75,7 \\
\hline Primaria & 1182 & 2,4 & 3,8 & 13,1 & 80,7 \\
\hline Secundaria & 1878 & 2,2 & 2,4 & 13,6 & 81,8 \\
\hline Superior & 979 & 0,8 & 1,9 & 11,4 & 85,9 \\
\hline \multicolumn{6}{|l|}{ Área } \\
\hline Urbano & 2611 & 1,2 & 1,5 & 12,4 & 84,9 \\
\hline Rural & 1564 & 4,3 & 6,8 & 14,8 & 74,1 \\
\hline \multicolumn{6}{|l|}{ Dominios } \\
\hline Costa norte & 576 & 5,3 & 2,7 & 7,4 & 84,6 \\
\hline Costa centro & 389 & 0,0 & 0,7 & 18,8 & 80,5 \\
\hline Costa sur & 266 & 0,0 & 1,0 & 16,4 & 82,6 \\
\hline Sierra norte & 297 & 5,9 & 1,8 & 8,8 & 83,5 \\
\hline Sierra centro & 885 & 0,5 & 0,7 & 8,9 & 89,9 \\
\hline Sierra sur & 490 & 3,5 & 15,9 & 32,6 & 48,0 \\
\hline Selva & 796 & 3,1 & 2,3 & 10,0 & 84,6 \\
\hline $\begin{array}{l}\text { Lima Metropo- } \\
\text { litana }\end{array}$ & 476 & 0,0 & 0,0 & 10,4 & 89,6 \\
\hline \multicolumn{6}{|l|}{ Pobreza } \\
\hline Pobre extremo & 303 & 5,5 & 3,5 & 8,1 & 82,8 \\
\hline Pobre & 883 & 2,4 & 2,5 & 13,5 & 81,5 \\
\hline No pobre & 2989 & 1,5 & 2,7 & 13,2 & 82,7 \\
\hline
\end{tabular}

Tabla 3. Medianas de yodo $(\mathrm{mg} / \mathrm{k})$ en sal según marcas mas consumidas en Cajamarca, Huancavelica, Huánuco, Loreto y San Martín; Perú 2012-2013

\begin{tabular}{lcccr}
\hline Marca de sal & Mediana & P25 & P75 & RIQ \\
\hline Marina & 40,1 & 30,7 & 48,1 & 17,4 \\
Pura Sal & 36,9 & 35,5 & 40,1 & 4,6 \\
Nor Sal & 36,2 & 34,7 & 40,0 & 5,4 \\
Norteño & 33,5 & 29,4 & 38,1 & 8,7 \\
Emsal & 32,6 & 28,3 & 39,4 & 11,1 \\
Sal y Mar & 29,5 & 21,2 & 35,6 & 14,4 \\
Cocinera & 21,2 & 17,0 & 28,4 & 11,4 \\
Mi Purita & 20,5 & 17,9 & 23,4 & 5,5 \\
Cris-Sal & 19,6 & 17,7 & 19,6 & 1,9 \\
Elita Sal & 19,0 & 15,3 & 19,8 & 4,5 \\
Otro & 16,5 & 12,8 & 21,4 & 8,6 \\
Salina & 13,8 & 10,2 & 18,4 & 8,3 \\
Peru Sal & 11,7 & 10,7 & 12,5 & 1,7 \\
Pirámide & 9,6 & 7,4 & 20,0 & 12,6 \\
\hline
\end{tabular}

mostró que la mediana de yodo según marcas de sal de consumo fluctúa entre 9,6 mg/k a 40,1 mg/k. La marca Marina tiene una mediana de 32,6 mg/k y Emsal de 40,1 $\mathrm{mg} / \mathrm{k}$. Se observó que las marcas Cris Sal, Elita Sal, Salina, Perú Sal y Pirámide Sal tuvieron medianas de yodo en sal por debajo de lo recomendado por la OMS.

\section{DISCUSIÓN}

Los resultados del estudio mostraron que la mediana de yoduria en las mujeres entre 12 a 49 años estuvo por encima de los valores adecuados, y disminuye según el grupo etario; estos resultados fueron ligeramente menores que lo reportado por Higa et al. ${ }^{(6)}$, aunque ambos estuvieron por encima de los valores normales, contrariamente a lo encontrado en mujeres australianas, cuya mediana de yoduria fue $56,0 \mu \mathrm{g} / \mathrm{L}{ }^{(21)}$ que está por debajo de lo recomendado por la OMS. Situación que se explica porque en Perú la yodación de la sal se realiza desde hace muchos años, por ende, se tiene dos fuentes de yodo, por un lado la sal yodada que se emplea en la preparación de los alimentos y, por otro lado, el consumo de alimentos naturales como el pescado, mariscos y vegetales como la cebolla y ajos que son ingredientes clave en las preparaciones que caracterizan a la gastronomía peruana, mientras que las mujeres australianas consumen frecuentemente alimentos procesados o industrializados que no necesariamente incluyen sal yodada ${ }^{(21)}$. Por otra parte, se observó una discreta tendencia descendente entre la edad y la mediana de yoduria, a medida que aumenta la edad disminuye la mediana de yoduria, pero los niveles se mantienen por encima de lo recomendado por la OMS, situación que podría explicarse por el consumo de alimentos procesados o preparaciones elaboradas 
fuera del hogar que son ricas en calorías y que están al alcance de las jóvenes, y adultas ${ }^{(22)}$.

Por otro lado, se observó cierta relación entre el nivel educativo y la mediana de yoduria, las mujeres analfabetas tuvieron una mediana muy cercana a los valores normales o adecuados, mientras que las alfabetas tuvieron medianas de yoduria por encima de los valores recomendados por la OMS, probablemente porque la mayoría de las mujeres están en los colegios o centros de trabajo y estarían consumiendo alimentos en los quioscos o cafeterías que frecuentemente expenden alimentos ricos en sal, por otra parte, la población peruana tienen cierta preferencia por el consumo de sal, al respecto, según Calderón et al. reportaron que el $97 \%$ de las mujeres en edad fértil consumen sal (22).

Los departamentos de Moquegua, Tacna, Madre de Dios y Ucayali tuvieron medianas de yoduria excesivos, lo cual podría indicar riesgo de consecuencias adversas de salud; mientras que Puno, Piura y Tumbes fueron los únicos departamentos que presentaron medianas de yoduria consideradas adecuadas u óptimas. Situación que resulta preocupante, porque conllevaría a pensar que existiría una ingesta excesiva de yodo en la dieta de las mujeres, y se sabe que los niveles excesivos de yodo en la dieta por largo tiempo terminan por producir bloqueo de la captación de yodo por la tiroides, lo que podría producir hiperproducción de TSH por la hipófisis que produce bocio y cáncer ${ }^{(23,24)}$.

El Centro Nacional de Alimentación y Nutrición, que realiza la evaluación de yodo en sal, en el marco de la vigilancia de la fortificación de la sal para consumo humano en plantas productoras a nivel nacional, reportó que las muestras evaluadas en Piura no estuvieron conformes (2009), pero en el año siguiente (2010), según la norma peruana, la evaluación de la muestras de sal estuvieron conformes (19). Al respecto, es necesario continuar con la vigilancia del cumplimiento de la ley, paralelamente, evaluar la ingesta de yodo en la población peruana e identificar las otras posibles fuentes de ingesta de yodo en la población peruana.

Llama la atención que $17,5 \%$ de la sal de consumo tenga niveles de yodo por debajo de 30 ppm, es preocupante que el $4,6 \%$ de las muestras tengan niveles de yodo en sal tan bajos (<7ppm), situación que es más frecuente en la sierra sur, la costa norte y la sierra norte, los resultados presentes constituyen casi el doble de lo reportado por Higa et al. a nivel nacional (6); sin embargo, no es posible realizar la comparación a nivel de dominios debido a que el diseño muestral del estudio en mención considera cinco dominios (Lima Metropolitana, resto de costa, sierra urbana, sierra rural y selva) mientras que la muestra de este estudio considera ocho dominios (Lima Metropolitana, costa norte, costa centro, costa sur, sierra norte, sierra centro, sierra sur, selva) estratificado por el Instituto Nacional de Estadística e Informática del Perú ${ }^{(15)}$.

Por otra parte, al evaluar la concentración de yodo en sal según marcas, se encontró que algunas marcas de sal tuvieron medianas de yodo en sal por debajo de $20 \mathrm{mg} / \mathrm{k}$, pese a que la legislación peruana establece el nivel de yodación entre 30 a 40 ppm, y la OMS establece que el nivel de yodación recomendado por la OMS-UNICEF-ICCIDD es de $20-40 \mathrm{mg}$ de yodo por kilo de sal, situación que podría explicarse por la condiciones de conservación y manejo de la sal en el hogar, lo cual podría disminuir la concentración de yodo en sal; asimismo, el uso de sal adulterada producida por empresas clandestinas que no cumplen con la normatividad peruana, por ello, la vigilancia del cumplimiento de la normatividad debería ser constante y periódica a nivel nacional ${ }^{(13)}$. Algunos estudios muestran que el clima influye en la cantidad de yodo en la sal, por ejemplo, en ambientes donde la humedad relativa es mayor de $76 \%$ la sal pierde más yodo comparado con ambientes con humedad relativamente menor $(25,26)$.

Los niveles de yodo en orina en las gestantes estuvo por encima de lo recomendado por la OMS, al igual que en las no gestantes, los resultados del presente estudio están por encima de lo encontrado en gestantes iraníes ${ }^{(27)}$ y españolas ${ }^{(28)}$; aunque la muestra de gestantes fue pequeña y los resultados deberían ser tomados como una aproximación de la situación real.

Una de las limitaciones del estudio fue la pérdida de algunas muestras de orina, que tuvieron que ser eliminadas porque no se encontraron viables para el análisis de cuantificación de yodo en la muestra, principalmente en las zonas rurales y más accidentadas del Perú en donde el mantenimiento y traslado de las muestras fueron afectadas por las condiciones climáticas, los desastres naturales, bloqueos de carretera, o conflictos sociales internos que se presentaron durante el periodo de estudio. Sin embargo, la cantidad de muestras obtenidas es considerable, lo cual no afectaría la estimación de los resultados con la inferencia de la muestra.

En conclusión, la mediana de yoduria en las mujeres entre 12 a 49 años a nivel nacional, está por encima de lo recomendado por la OMS y la mayoría de las muestras de sal tuvieron concentraciones adecuadas de yodo según la OMS. Los departamentos de Moquegua, Tacna, Madre de Dios y Ucayali tuvieron niveles excesivos de yoduria. Se sugiere a las autoridades sanitarias 
la implementación de programas o intervenciones educativas en la población general para promover el adecuado consumo de alimentos fuentes de yodo y sal yodada. Asimismo, se sugiere el trabajo articulado entre los sectores de salud, pesquero y educación para promover el consumo de especies marinas, alimentos fuentes de yodo y sal yodada mediante campañas informativas dirigidas a la población en general, en especial a los estudiantes de colegios públicos y privados, por otra parte, es conveniente mantener la vigilancia de la yodación de sal en las plantas de producción de sal a nivel nacional para garantizar los niveles adecuados de yodo en sal de consumo humano.

Agradecimientos: al equipo de supervisión, bioquímica y antropometría de la Dirección Ejecutiva de Vigilancia
Alimentario y Nutricional y la Dirección Ejecutiva de Ciencia y Tecnología de Alimentos del Centro Nacional de Alimentación y Nutrición del INS.

Contribuciones de autoría: CTM, ha participado en la concepción del artículo, procesamiento de datos, análisis y redacción de la versión final. JRSA, DAD, TJL, IFT participaron en la redacción, todos los autores aprobaron la versión final.

Fuentes de financiamiento: este estudio fue financiado por el Instituto Nacional de Salud y el recojo de datos se realizó en la muestra de hogares del INEI, en el marco del convenio suscrito entre el Instituto Nacional de Estadística e Informática y el Instituto Nacional de Salud.

Conflictos de interés: los autores declaran que no existe conflicto de interés en la ejecución o publicación de este estudio.

\section{REFERENCIAS BIBLIOGRÁFICAS}

1. Caballero L. Yoduria en escolares y embarazadas del estado Trujillo, Venezuela 2007-2008. Rev Argent Endocrinol. Metab. 2011;28(4):206-11.

2. Hetzel BS. Progress in the prevention and control of iodine deficiency disorders. Lancet. 1987 Aug 1;2(8553):266.

3. Bleichrodt N, Born MP. A metaanalysis of research on iodine and its relationshipto cognitive development. In: Stanbury JB, ed. The damaged brain of iodine deficiency. New York, NY: Cognizant Communication; 1994. p. 195200.

4. Vejbjerg P, Knudsen N, Perrild H, Laurberg P, Carlé A, Pedersen IB, et al. Lower prevalence of mild hyperthyroidism related to a higher iodine intake in the population: prospective study of a mandatory iodization programme. Clin Endocrinol (Oxf). 2009 Sep;71(3):440-5.

5. Gallego M, Loango N, Londoño A, Landazuri P. Niveles de excreción urinaria de yodo en escolares del Quindío, 2006-2007. Rev Salud Publica (Bogota). $2009 \mathrm{Dec} ; 11(6): 952-60$

6. Higa AM, Miranda M, Campos M, Sánchez JR. Ingesta de sal yodada en hogares y estado nutricional de yodo en mujeres en edad fértil en Perú, 2008. Rev Peru Med Exp Salud Publica. 2010 Jun;27(2):195-200.

7. Miranda M, Chávez H, Aramburú A, Tarqui-Mamani C. Estado nutricional de yodo en alumnos de escuelas públicas del nivel primario en el Perú, 2009.
[Internet]. Lima: Instituto Nacional de Salud; 2011 [citado el 17 de marzo de 2014]. Disponible en: http://www. ins.gob.pe/repositorioaps/0/5/zop/ zona izquierda 1/INFORME\%20 DDI\%202009\%20v2.pdf

8. Bauch K, Meng W, Ulrich FE, Grosse E, Kempe R, Schönemann F, et al. Thyroid status during pregnancy and post partum in regions of iodine deficiency and endemic goiter. Endocrinol Exp. 1986 Mar;20(1):67-77.

9. Caulfield LE, Richard SA, Rivera JA, Musgrove P, Black RE. Stunting, wasting, and micronutrient deficiency disorders. In: Jamison DT, Breman JG, Measham AR, Alleyne G, Claeson M, Evans DB, et al. (eds). Disease Control Priorities in Developing Countries. 2nd ed. New York: Oxford University Press; 2006. p. 551-68.

10. Enriching Lives: Overcoming vitamin and mineral malnutrition in developing countries. Washington, D.C.: Banco Mundial; 1994.

11. World Health Organization, United Nations Children's Fund, and the International Council for the Control of Iodine Deficiency Disorders. Global prevalence of iodine deficiency disorders. Micronutrient Deficiency Information System Working Paper 1. Geneva, Switzerland: WHO; 1993.

12. United Nations Children's Fund. The State of the World's Children 2012: Children in an Urban World. New York: United Nations Children's Fund (UNICEF); 2012.
13. World Health Organization (WHO), United Nations Children's Fund (UNICEF), International Council for Control of Iodine Deficiency Disorders (ICCIDD). Assessment of iodine deficiency disorders and monitoring their elimination. A guide for programme managers. 3rd ed. WHO/UNICEF/ ICCIDD. Geneva: WHO; 2008.

14. Ministerio de Salud. Informe de la evaluación de la situación de desórdenes de deficiencia de yodo. Lima, Perú: Ministerio de Salud; 1996.

15. Instituto Nacional de Estadística e Informática (INEI). Evolución de la pobreza monetaria 2009-2013. Lima: INEI; 2014

16. Pino S, Fang SL, Braverman LE. Ammonium persulfate:a safe alternative oxidizing reagent for measuring urinary iodine. Clin Chem. 1996 Feb;42(2):239-43.

17. Dunn JT, Crutchfield HE, Gutekuntz R, Dunn AD. Methods for measurement iodine in urine. The Netherlands: International Council for control of iodine deficiency disorders ICCIDDUNICEF-WHO; 1993.

18. World Health Organization. Salt reduction and iodine fortification strategies in public health [Internet]. Australia: OMS, 2013; [citado 22 Mar 2015]. Disponible en: http://apps.who.int/iris/bitstre $\mathrm{am} / 10665 / 101509 / 1 / 978924150669$ 4_eng.pdf?ua=1

19. Valdivia S, Robles S, Ramirez G. Vigilancia de la fortificacion de la sal para consumo 
humano en el Perú periodos 2009-2010. [internet]. Lima: Instituto Nacional de Salud; 2011. [citado 09 Jul 2014]. Disponible en: http://www.ins.gob.pe/repositorioaps/0/5/ jer/VIGI_SAL_CH/Inf_Tec_Sal_030812. pdf

20. Ministerio de Salud. Eliminación de los desórdenes por deficiencia de yodo en el Perú. Lima, Perú: Ministerio de Salud; 2003.

21. Charltoti KE, Yeatman HR, Houweling F. Poor iodine status and knowledge related to iodine on the eve of mandatory iodine fortification in Australia. Asia Pac J Clin Nutr. 2010;19(2):250-5.

22. Calderón M, Moreno C, Rojas C, Barboza J. Consumo de alimentos según condición de pobreza en mujeres peruanas en edad fértil y niños menores de 12 a 35 meses de edad. Rev Peru Med Exp Salud Publica. 2005;22(1):19-25.

23. Wolff J. Iodide goitre and the pharmacological effects of excess iodide. Am J Med. 1969 Jul; $47(1): 101-24$.

24. Glantre E, Haldorsen T, Berg JP, Stensvold I, Solvoll K. Norwegian case-control study testing the hypothesis that seafood increases the risk of thyroid cancer. Cancer Causes Control. 1993 Jan;4(1):11-6.

25. Biber FZ, Unak P, Yurt F. Stability of iodine content in iodized salt. Isotopes Environ Health Stud. 2002 Jun;38(2):87-93.

26. Wang GY, Zhou RH, Wang Z, Shi L, Sun M. Effects of storage and cooking on the iodine content in iodized salt and study on monitoring iodine content in iodized salt. Biomed Environ Sci. 1999 Mar;12(1):1-9.

27. Rostami R, Beiranvand, A Khakhali $\mathrm{H}$, Salary S, Aghasi M, Nourooz-Zadeh J. Evaluation of Accessibility of Iodinated Salt and Nutritional Iodine Status during Pregnancy. Iran J Public Health. 2012;41(8):56-60.

28. Alvarez-Pedrerol M, Ribas-Fitó N, García-Esteban R, Rodriguez A, Soriano D, Guxens $\mathrm{M}$, et al. Iodine sources and iodine levels in pregnant women from an area without known iodine deficiency. Clin Endocrinol (Oxf). 2010 Jan;72(1):81-6.

Correspondencia: Carolina Tarqui Mamani Dirección: Instituto Nacional de Salud Calle Tizón y Bueno 276; Jesús María. Lima, Perú

Correo electrónico:carobtm@gmail.com

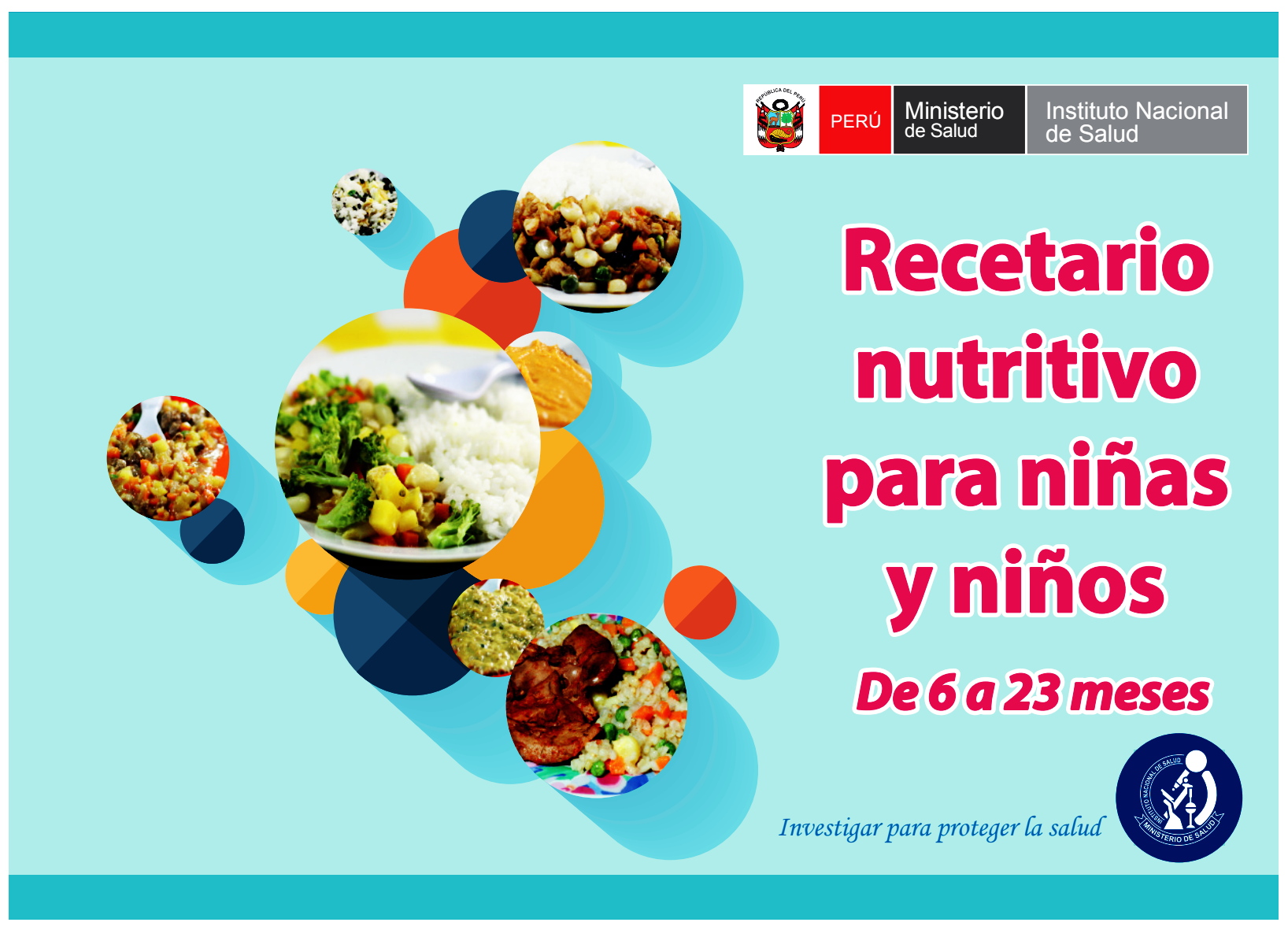

